Conclusions The impairment in respiration and hearing which is higher among the workers exposed to dust, fumes and noise at the workplace shows a need for future research to study if all safety measures are strictly adhered to. The impairment in near vision which was higher among the exposed workers needs detailed investigations into the cause and association with the working environment, if any.

\section{P-100 MEDICAL ACCESSIBILITY AND UNDER-REPORTING OF OCCUPATIONAL DISEASES: EFFECT OF TRAVEL DISTANCE AND TRAVEL TIME}

${ }^{1}$ Ping Hui Chen, Pau Chung Chen. 'National Taiwan University Hospital Hsinchu Branch, Taiwan

\subsection{6/OEM-2021-EPI.199}

Introduction In Taiwan, outpatients' average travel distance (TD) is $17.68 \mathrm{~km}$, and workers' TD and travel time (TT) for outpatient services are only $8.2 \mathrm{~km}$ and 27.6 mins. Poor medical accessibility of occupational outpatient service can also lead to under-reporting of occupational diseases (ODs).

Methods In Taiwan, Network of Occupational Diseases and Injuries Service (NODIS), composed of 9 major reporting hospitals, is an important surveillance system of ODs. Using NODIS's reporting data and manpower survey from 2008 to 2018, we calculate each town's incidence rate of occupation diseases (IROD) and expected IROD according to workers' occupations and job titles, and each town's shortest TD and TT to 9 major reporting hospitals is estimated by Google Maps' Distance Matrix API. Quasi-Poisson regression model is employed to investigate effect of TD and TT on IROD.

Results There are 8017 cases of suspected ODs in NODIS from 2008 to 2018, and 3306 cases are confirmed as definite ODs. Adjusted by workers' occupations and job titles, as TD/ TT increases by $10 \mathrm{~km} / 10$ mins, IROD significantly decreases by $10.90 \% / 10.74 \%$, and less-disabled workers who have never stopped working or lost their jobs are more impeded by long TD and TT.Compared with towns with TD $45 \mathrm{~km}$ and offshore towns decreases by 38.93\%, 39.58\%, 50.03\%, 55.01\%, $65.71 \%$, and $84.10 \%$, and IRODs of towns with TT 10-15, $15-25,25-35,35-45,>45$ mins and offshore towns decreases by $30.94 \%, 43.57 \%, 47.41 \%, 47.70 \%, 67.81 \%$, and $85.29 \%$. Around $40 \%$ ODs are under-reported due to poor medical accessibility.

Conclusion Our study shows how poor medical accessibility leads to serious under-reporting, and up to $40 \%$ ODs could be under-reported. Using this method, we can identify areas with poor medical accessibility and evaluate cost-effectiveness of adding reporting hospital.

\section{P-101 OCCUPATIONAL DUST EXPOSURES AND CT FINDINGS OF INTERSTITIAL LUNG DISEASE AND CHRONIC OBSTRUCTIVE PULMONARY DISEASE}

${ }^{1}$ Inge Brosbøl Iversen, Kennet Sønderstgaard Thorup, Jesper Thygesen, Finn Rasmussen, Michael Brun Andersen, Elisabeth Bendstrup, Zara Ann Stokholm, Else Toft Würtz, Vivi Schlünssen, Jens Peder Ellekilde Bonde, Jakob Hjort Bønløkke, Hans Kromhout, Henrik Al. ${ }^{1}$ Aarhus University Hospital, Denmark

10.1136/OEM-2021-EPI.200
Introduction Occupational dust exposure is associated with interstitial lung disease and chronic obstructive pulmonary disease, but little is known about the association with more discrete lung changes detected by lung scans.

Objectives To 1) analyze the relation between occupational dust levels and HRCT (high-resolution computed tomography) detected signs of pulmonary disease, and 2) map the prevalence of these signs in the Danish workforce.

Methods We are carrying out a cross-sectional study of 25,000 adults who underwent HRCT scans of the lungs 2011-2019 in Denmark. We will analyze the extent of emphysema and signs of pulmonary fibrosis such as, but not limited to, reticulation with Imbio Lung Texture AnalysisTM of the HRCT scans. The DOC*X cohort provides annual information on occupation (ISCO-88) and industry for the total Danish workforce since 1976. Individual exposure levels are estimated using quantitative job exposure matrices for asbestos, crystalline silica, wood dust, and endotoxins. We will conduct adjusted analyses of exposure-response relations and tabulate distributions of emphysema and signs of pulmonary fibrosis for all occupations and industries.

Conclusion The study will provide new knowledge on pulmonary effects of current and past occupational dust levels. We will use a new software for objective identification and quantification of signs of pulmonary disease independent of diagnostic traditions. This sensitive and graduated measure of outcome will also enable more sensitive exposure-response analyses that include discrete signs of pulmonary disease. We expect this study to serve as a basis for targeted interventions of importance to the many that still have dusty work.

\section{P-108 POPULATION-LEVEL ESTIMATES OF OCCUPATIONAL EXPOSURE TO CHLOROTHALONIL, 2,4-D, AND GLYPHOSATE IN CANADA'S AGRICULTURAL INDUSTRY (CAREX CANADA)}

${ }^{1}$ Ela Rydz, Cheryl Peters, Kristian Larsen. 'Simon Fraser University, Canada

\subsection{6/OEM-2021-EPI.201}

Introduction Certain pesticides may lead to adverse health outcomes including cancer; however, little is known about occupational pesticide exposure in Canada.

Objective The purpose of this study was to estimate the prevalence and likelihood of occupational exposure to chlorothalonil, 2,4-D, and glyphosate in Canada's agricultural industry.

Methods Lower and upper estimates were calculated using the Canadian Census of Population (CoP) and Census of Agriculture $(\mathrm{CoA})$. We estimated the number of workers and proportion of farms applying 'herbicides' or 'fungicides' by farm type using CoA survey data. These values were multiplied to yield the number of workers at risk of exposure. Likelihood of exposure (exposed, probably exposed, possibly exposed) was qualitatively assigned using information on crop type, primary expected tasks, crop production practices, and residue transfer data. Agricultural workers who are at risk of exposure but were not captured by the CoA were identified using the CoP.

Results An estimated 37,700 to 55,800 workers (11-13\% of agricultural workers) were exposed to glyphosate in in Canada while 30,800 to 43,600 workers $(9-11 \%)$ and 9,000 to $14,100(3 \%)$ were exposed to $2,4-\mathrm{D}$ and chlorothalonil, 
respectively. Approximately $70-75 \%$ of at-risk workers were probably or possibly exposed to any of the pesticides. Glyphosate exposure was most common among workers in oilseed (29\%) and dry pea/bean farms (28\%), along with those providing support activities for farms (31\%). 2,4-D exposure was most common in corn (28\%), other grain $(28 \%)$, and soybean farms (27\%), while chlorothalonil exposure was more likely among greenhouse, nursery and floriculture workers (42\%) and those working on farms (28\%, for occupations not captured by the CEAG). Regional variations reflected differences in farm types by province.

Conclusion This study estimated the prevalence of occupational pesticide exposure in Canada, and findings can support priority setting for future research and data collection.

\section{P-113 AN EXPLORATION OF DISPENSING ERRORS AMONG THE HOSPITAL PHARMACISTS IN TAIWAN}

${ }^{1}$ Kuan-Han Lin, Yun-Chia Chang. ${ }^{~}$ Asia University, Taiwan

\subsection{6/OEM-2021-EPI.202}

Introduction Dispensing errors are among the most common medical errors. Previous studies found that environmental factors, educational training, physical and mental health were factors affecting dispensing errors among pharmacists. However, the associations among burnout, sleep quality and dispensing error in pharmacists are still unknown.

Objectives The aims of this study were to investigate the frequency of dispensing errors among the hospital pharmacists in Taiwan and to determine the risk factors associated with it.

Methods This was a cross-sectional study. The electronic questionnaires were sent to the pharmacists from multiple centers. The questionnaire included demographic characteristics, the frequencies of dispensing errors in the past three months, environmental factors, Chinese version of Copenhagen Burnout Inventory, Chinese version of Pittsburgh Sleep Quality Index, and continuing education questions. Logistic regression models were used to assess the association between environmental factors, burnout, sleep quality, continuing education and dispensing errors.

Results A total of 370 pharmacists completed the questionnaire. Among them, 96.5\% reported dispensing errors in the past three months. In the univariate logistic analysis, pharmacists experienced dispensing errors were more likely to have shorter working years (Odds Ratio $(\mathrm{OR})=0.91$, 95\% Confidence Interval (CI) : 0.85-0.98), shift work (OR=4.75, 95\% CI : 1.51-14.89), shorter working hour per day $(\mathrm{OR}=0.80$, 95\% CI: 0.64-0.99), lower personal burnout score $(\mathrm{OR}=$ 0.97, 95\% CI: 0.94-0.99) and lower over-commitment score $(\mathrm{OR}=0.96$, 95\% CI: 0.94-0.99) compared with those without dispensing errors. The multivariate logistic regression model indicated that after adjusting for all possible risk factors, shorter working years (adjusted Odds Ratio $(\mathrm{aOR})=$ 0.92, 95\% CI: 0.85-0.99) and shift work (aOR $=4.63,95 \%$ CI: 1.39-15.45) was associated with dispensing errors among pharmacists.

Conclusion A significant proportion of pharmacists experienced dispensing errors. Development of stress management programs to enhance pharmacists' physical and psychological wellbeing and to improve job performance is warranted.

\section{P-115 PRO-MENTA: IMPACT OF MAJOR ORGANIZATIONAL CHANGES ON EMPLOYEE MENTAL HEALTH AND WORKPLACE PRODUCTIVITY}

'Lea Nørgaard Sørensen, Morten Vejs Willert, Johan Høy Jensen, Vita Ligaya Dalgaard, Marianne Kyndi. 'Aarhus University Hospital, Denmark

\subsection{6/OEM-2021-EPI.203}

Introduction The health care system of Denmark is currently undergoing major centralizations. Relocation of workplaces as well as mergers and split-ups may impact both health and productivity among employees and managers. The unification of all hospital units in Aarhus, Denmark, poses an opportunity to study the short- and long-term consequences of different degrees of organizational change during a fixed event.

Objectives The primary aims are to: 1) investigate the consequences of major organizational changes on absenteeism and mental health of employees and workplace productivity and staff turnover, and 2) identify risk factors for negative consequences on mental health, sickness absence, productivity, and staff turnover, in order to identify risk groups for targeted prevention.

Methods Using national and regional registers we will establish a cohort of 10,000 individuals employed at Aarhus University Hospital in the period from 2011-2020. Hospital units moving at different time points through 2016-2019 will be aligned on the index date defined by their relocation and followed up to 5 years after and prior to this.

Results Based on the life event literature we expect to find a delayed effect of major organisational change on sickness absence and mental health, and an immediate effect on staff turnover and productivity. We hypothesize these effects as more pronounced for units which in addition to relocating also merge/split, representing greater organisational change. We also expect to identify risk factors for the chosen outcomes based on job titles, age, seniority, and work unit characteristics such as number of employees, patient centred vs. service/ administrative, and out-patient vs. in-patient wards.

Conclusion This project is expected to produce valuable knowledge about the mental health and/or economic consequences of major organizational changes in the health care system. This can inform preventive actions targeting specific employee groups in future centralization projects.

\section{P-118 MANAGEMENT OF CAUSAL UNCERTAINTY : CAN A SICK BUILDING SYNDROM BECOME CHRONIC ?}

${ }^{1}$ Christine Meffre, Yoann Dominique, Juliette Chatelot, Eve Bourgkard, Michel Vernay. ${ }^{1}$ Agence Nationale de Santé Publique, France

\subsection{6/OEM-2021-EPI.204}

Objectives In 2014, the head of a elderly care facility (ECF) alerted the regional health authority for chronic irritative symptoms among office workers. Symptoms started in 2009 for 4 workers back in a newly rehabilitated building. From 2009 to 2014, the first analysis of indoor air quality lead to cleaning and disinfection of the air treatment plant. Furthermore no toxic compounds were identified in the materials used. In 2014, out of 21 office workers, 13 declared irritative symptoms, and the French national public health agency was called to carry out epidemiological investigations. 